\title{
Disparate effects of pharmacotherapy on plasma plasminogen activator inhibitor-1 levels in women with the polycystic ovary syndrome
}

\author{
Ekaterini Koiou, ${ }^{1}$ Konstantinos Tziomalos, ${ }^{2}$ Ilias Katsikis, ${ }^{1}$ Dimitrios Delkos, ${ }^{1}$ \\ Elena A. Tsourdi, ${ }^{1}$ Dimitrios Panidis ${ }^{1}$
}

${ }^{1}$ Division of Endocrinology and Human Reproduction, Second Department of Obstetrics and Gynecology, Aristotle University of Thessaloniki, Hippokration Hospital, ${ }^{2}$ First Propedeutic Department of Internal Medicine, Aristotle University of Thessaloniki, AHEPA Hospital, Thessaloniki, Greece

\begin{abstract}
OBJECTIVE: Polycystic ovary syndrome (PCOS) is characterized by obesity and insulin resistance (IR), which result in elevated plasminogen activator inhibitor-1 (PAI-1) levels. We aimed to assess the changes in PAI-1 levels in PCOS during treatment with metformin and during weight loss. DESIGN: Twenty-three normal weight women with PCOS were given metformin $850 \mathrm{mg}$ bid for 6 months. Fifty overweight/obese women with PCOS were prescribed an energy-restricted diet, were instructed to exercise and were randomized to orlistat $120 \mathrm{mg}$ tid or sibutramine $10 \mathrm{mg}$ qd for 6 months. RESULTS: In normal weight women, treatment with metformin reduced the body mass index (BMI) and circulating androgens, improved markers of IR and lowered PAI-1 levels. In overweight/obese women, sibutramine and orlistat yielded comparable reductions in BMI and markers of IR. In contrast, the effects on the free androgen index (FAI) differed $(p=0.027)$ : sibutramine reduced the FAI $(p=0.005)$, whereas orlistat had no effect. The effects of sibutramine and orlistat on PAI-1 levels also differed $(p=0.042)$ : sibutramine reduced PAI-1 levels $(p<0.001)$, whereas orlistat had no effect. CONCLUSIONS: Metformin and sibutramine, but not orlistat, reduce PAI-1 levels in PCOS. The reduction in circulating androgens during metformin and sibutramine treatment might be implicated in this decline.
\end{abstract}

Key words: Hyperandrogenemia, Insulin resistance, Metformin, Orlistat, Plasminogen activator inhibitor-1, Polycystic ovary syndrome, Sibutramine, Weight loss

Address for correspondence:

Konstantinos Tziomalos, MD, PhD, First Propedeutic

Department of Internal Medicine, AHEPA Hospital,

1 Stilponos Kyriakidi Str., 546 36, Thessaloniki, Greece,

Tel.: +30 2310994621, Fax: + 302310274434 ,

e-mail: ktziomalos@yahoo.com

Received 06-03-2012, Accepted 15-06-2012

\section{INTRODUCTION}

The polycystic ovary syndrome (PCOS) is a common endocrine disorder that shows substantial phenotypic variability. ${ }^{1}$ Insulin resistance (IR) and abdominal obesity are two of the major characteristics of PCOS, being present in $50-70 \%$ and $50-60 \%$ of women with 
the syndrome, respectively. ${ }^{1}$ Even though IR is observed in the majority of overweight/obese women with PCOS, it is also found in normal weight women with the syndrome, suggesting that IR represents an intrinsic endocrine abnormality in this disorder. ${ }^{2,3}$

Plasminogen activator inhibitor-1 (PAI-1) is a major regulator of fibrinolysis. ${ }^{4}$ In addition, plasma PAI-1 antigen levels and activity are frequently elevated in both obese and insulin-resistant subjects. ${ }^{4}$ Interestingly, most studies in women with PCOS have reported that plasma PAI-1 antigen levels are increased in both normal weight and overweight/ obese women with PCOS compared with body mass index (BMI)-matched controls. ${ }^{5-7}$ More importantly, elevated plasma PAI-1 levels are associated with increased risk for both type 2 diabetes mellitus (T2DM) and cardiovascular disease (CVD), ${ }^{8,9}$ i.e. disorders that appear to be prevalent in women with PCOS. ${ }^{10}$ This association between PAI-1 levels and T2DM is explained in part by the relationship between PAI-1 levels and both IR and abdominal obesity, which are major risk factors for T2DM. ${ }^{4,8}$ In addition, PAI- 1 is partly produced in endothelial cells and represents a marker of endothelial dysfunction, which in turn also appears to increase the risk for T2DM by decreasing blood flow in the skeletal muscle, resulting in IR.,8

Given the potential role of obesity and IR in the pathogenesis of PCOS, weight loss is a first-line treatment option for overweight/obese women with PCOS. ${ }^{11}$ Metformin, an insulin-sensitizing agent, is frequently administered to both overweight/obese and normal weight women with PCOS. ${ }^{11}$ However, there are very limited data on the effects of weight loss and metformin on plasma PAI-1 levels in women with PCOS. ${ }^{12}$ Accordingly, the aim of the present study was to assess the changes in plasma PAI-1 levels during a) treatment with metformin of normal weight patients with PCOS and b) during diet, exercise and pharmacotherapy (with orlistat or sibutramine) in overweight/obese patients with this syndrome.

\section{PATIENTS AND METHODS}

\section{Patients}

We studied 23 normal weight women with PCOS [mean age 19.9 \pm 3.1 years, mean body mass index (BMI) $\left.22.1 \pm 2.2 \mathrm{~kg} / \mathrm{m}^{2}\right]$ and 50 overweight/obese women with the syndrome (age 25.7 \pm 5.9 years, BMI $33.5 \pm 5.2 \mathrm{~kg} / \mathrm{m}^{2}$ ), who were being followed up at the Gynecological Endocrinology Infirmary of the Second Department of Obstetrics and Gynecology, Aristotle University of Thessaloniki. The study was performed between January of 2007 and December of 2009.

Diagnosis of PCOS was based on the revised criteria of Rotterdam. ${ }^{13}$ None of the women studied had galactorrhea or any endocrine or systemic disease that could possibly affect reproductive physiology. A Synacthen test was performed with tetracosactide (Synacthen $0.25 \mathrm{mg} / 1 \mathrm{ml}$; Novartis Pharma, RueilMalmaison, France) in each woman with a basal $17 \alpha$-hydroxyprogesterone (17 $\alpha$-OHP) plasma level $>1.5 \mathrm{ng} / \mathrm{ml}$ to exclude congenital adrenal hyperplasia.

No woman reported use of any medication that could interfere with the normal function of the hypothalamic-pituitary-gonadal axis during the last semester. Informed consent was obtained from all women and the study was approved by the Institutional Review Board; the study met the requirements of the 1975 Helsinki guidelines.

\section{Study protocol}

In all women, weight, height and waist circumference $(\mathrm{W})$ were measured as described previously. ${ }^{14,15}$ Baseline blood samples were collected between days 3 and 7 of the menstrual cycle in the control group and after a spontaneous bleeding episode in the PCOS group, after an overnight fast. The circulating levels of follicle-stimulating hormone (FSH), luteinizing hormone (LH), prolactin (PRL), total testosterone $(\mathrm{T}), \Delta 4$-androstenedione $(\Delta 4-\mathrm{A})$, dehydroepiandrosterone-sulfate (DHEA-S), $17 \alpha-\mathrm{OHP}$, sex hormonebinding globulin (SHBG), glucose and insulin were measured. Immediately after the baseline blood sampling, an oral glucose tolerance test (OGTT) was performed in all patients; $75 \mathrm{~g}$ of glucose were administered orally and serum glucose levels were determined after 30, 60, 90 and $120 \mathrm{~min}$. On the same day transvaginal ultrasonography was performed and the volume of each ovary was determined as well as the number of follicles in each ovary.

The normal weight women with PCOS were treated with metformin $850 \mathrm{mg}$ bid for 6 months. ${ }^{14,15}$ All baseline laboratory tests were repeated at the end of treatment with metformin (i.e. at 6 months). 
In overweight/obese women with PCOS, the basal metabolic rate (in $\mathrm{kcal} /$ day) was calculated at baseline and adjusted for moderate daily physical activity as follows: in women $18-30$ years of age: $(0.0621 \mathrm{x}$ weight in $\mathrm{kg}+2.0357) \times 240 \times 1.3$ and in women $\geq 31$ years of age: $(0.0342 \mathrm{x}$ weight in $\mathrm{kg}+3.5377) \mathrm{x} 240 \mathrm{x} 1.3$. All overweight/obese women were prescribed a normalprotein, energy-restricted diet [basic metabolic rate - $600 \mathrm{kcal} /$ day, consisting of $50 \%$ from carbohydrate, $30 \%$ from fat (10\% saturated) and $20 \%$ from protein] and were instructed to exercise (moderate intensity aerobic exercise) for at least 3 hours/week for a period of 6 months. Overweight/obese women were also randomized to receive orlistat $120 \mathrm{mg}$ tid before each meal $\left(X_{\text {Xenical }}{ }^{\circledR}\right.$, Roche [Hellas] S.A., Greece) $(\mathrm{n}=22)$ or sibutramine $10 \mathrm{mg}$ qd in the morning (Reductil ${ }^{\circledR}$, Abbot Hellas, Greece) $(n=28)$ for 6 months. ${ }^{14,15}$ All baseline laboratory tests were repeated at the end of treatment with orlistat or sibutramine combined with diet and exercise (i.e. at 6 months).

The OGTT and the transvaginal ultrasonography were repeated at 6 months in overweight/obese women with PCOS but not in normal weight women with PCOS.

\section{Methods}

Serum levels of glucose, insulin, FSH, LH, PRL, androgens and $17 \alpha-\mathrm{OHP}$ were determined as described previously. ${ }^{16}$ Plasma PAI-1 levels were determined with an enzyme-linked immunosorbent assay (Bender MedSystems GmbH, Vienna, Austria). Free androgen index (FAI) was determined as follows: $\mathrm{FAI}=\mathrm{T}(\mathrm{nmol} / \mathrm{l}) \times 100 / \mathrm{SHBG}(\mathrm{nmol} / \mathrm{l}) \cdot{ }^{17} \mathrm{The}$ homeostasis model assessment of IR (HOMA-IR) index was calculated as follows: HOMA-IR =insulin $(\mu \mathrm{IU} / \mathrm{ml}) \times$ glucose $(\mathrm{mg} / \mathrm{dl}) / 405 .{ }^{18}$ The Quantitative insulin sensitivity check index (QUICKI) was calculated as follows: QUICKI $=1 /[\log I n s u l i n(\mu I U / m l)$ $+\log$ Glucose $(\mathrm{mg} / \mathrm{dl})] \cdot{ }^{19}$

\section{Transvaginal ultrasonography}

Transvaginal ultrasound scans of the ovaries were performed by an experienced sonographer in women who participated in the study. Ovarian volume was calculated by the formula $\mathrm{V}=(\pi / 6) \times D_{\text {length }} \mathrm{x} D_{\text {width }} \mathrm{X}$ $D_{\text {thickness, }}$ where $D$ is dimension. The presence of polycystic ovaries was diagnosed by the presence of 12 or more follicles in each ovary measuring 2-9 $\mathrm{mm}$ in diameter and/or increased ovarian volume $\left(>10 \mathrm{~cm}^{3}\right)$.

\section{Statistical analysis}

Data analysis was performed with the statistical package SPSS (version 17.0; SPSS Inc., Chicago, IL). Data are reported as mean \pm SD.

At baseline, correlations between plasma PAI-1 levels and other parameters were assessed by Pearson's correlation coefficient. Parameters that correlated significantly with plasma PAI-1 levels according to Pearson's correlation coefficient were included in a stepwise linear regression analysis model to assess for independent correlations with plasma PAI-1 levels. Differences at baseline between overweight/obese women treated with sibutramine and women treated with orlistat were assessed with the independent samples t-test. Changes between baseline and endof-treatment in normal weight women were assessed with the paired samples t-test. Changes between baseline and end-of-treatment in overweight/obese women were assessed with 2-way repeated measures analysis of variance via the Holm-Sidak method for multiple comparison testing. A general linear model for repeated measures was used to compare the changes in plasma PAI-1 levels between the three treatments (metformin, sibutramine and orlistat) after adjusting for the change in BMI. In all cases, a $p$ value $<0.05$ was considered significant.

\section{RESULTS}

In normal weight women with PCOS, plasma PAI1 levels at baseline correlated in univariate analysis with the BMI $(r=0.602, p=0.002)$ and serum FSH levels $(r=-0.499, p=0.015)$. In multivariate regression analysis, plasma PAI-1 levels were independently correlated with the BMI $(\mathrm{p}=0.002)$, after adjusting for serum FSH levels.

Changes in normal weight women with PCOS after 6 months of metformin treatment are shown in Table 1. A significant decrease in BMI was observed $(p=0.009)$. There was also a reduction in circulating androgens (serum T levels and FAI) and an improvement in markers of IR (serum glucose levels and QUICKI). Treatment with metformin also induced a significant decrease in plasma PAI-1 levels $(\mathrm{p}<0.001)$. 
Table 1. Changes in normal weight women with the polycystic ovary syndrome after 6 months of metformin treatment

\begin{tabular}{|c|c|c|c|}
\hline & Baseline & Month 6 & $\mathbf{p}$ \\
\hline BMI $\left(\mathrm{kg} / \mathrm{m}^{2}\right)$ & $22.1 \pm 2.2$ & $21.4 \pm 2.1$ & 0.009 \\
\hline FSH (mIU/ml) & $6.1 \pm 1.8$ & $6.4 \pm 3.3$ & 0.618 \\
\hline LH (mIU/ml) & $11.4 \pm 6.9$ & $13.0 \pm 13.9$ & 0.636 \\
\hline Prolactin (ng/ml) & $15.4 \pm 6.8$ & $14.6 \pm 7.4$ & 0.631 \\
\hline Testosterone (ng/dl) & $82.9 \pm 17.5$ & $72.9 \pm 16.8$ & 0.036 \\
\hline$\Delta 4-\mathrm{A}(\mathrm{ng} / \mathrm{ml})$ & $3.1 \pm 0.8$ & $2.9 \pm 0.8$ & 0.422 \\
\hline DHEA-S (ng/ml) & $3320.8 \pm 800.4$ & $3553.8 \pm 1152.3$ & 0.325 \\
\hline FAI & $8.52 \pm 4.08$ & $6.53 \pm 2.69$ & 0.030 \\
\hline $17 \alpha-\mathrm{OHP}(\mathrm{ng} / \mathrm{ml})$ & $1.2 \pm 0.5$ & $1.4 \pm 0.9$ & 0.196 \\
\hline SHBG (nmol/l) & $40.4 \pm 18.8$ & $43.4 \pm 15.3$ & 0.262 \\
\hline Glucose (mg/dl) & $98.8 \pm 16.6$ & $86.5 \pm 7.0$ & $<0.001$ \\
\hline Insulin $(\mu \mathrm{IU} / \mathrm{ml})$ & $18.7 \pm 31.7$ & $10.5 \pm 9.7$ & 0.140 \\
\hline Glucose/insulin & $10.02 \pm 5.35$ & $12.45 \pm 6.80$ & 0.147 \\
\hline HOMA-IR & $4.43 \pm 6.69$ & $2.24 \pm 2.05$ & 0.070 \\
\hline QUICKI & $0.33 \pm 0.03$ & $0.35 \pm 0.03$ & 0.009 \\
\hline PAI-1 (ng/ml) & $39.9 \pm 12.3$ & $28.9 \pm 8.6$ & $<0.001$ \\
\hline
\end{tabular}

BMI, body mass index; FSH, follicle stimulating hormone; $\mathrm{LH}$, luteinizing hormone; $\Delta 4$-A, $\Delta 4$-androstenedione; DHEAS, dehydroepiandrosterone sulfate; FAI, free androgen index; $17 \alpha-\mathrm{OHP}$, $17 \alpha$-hydroxyprogesterone; SHBG, sex hormone-binding globulin; HOMA-IR, homeostasis model assessment of insulin resistance; QUICKI, quantitative insulin sensitivity check index; PAI-1, plasminogen activator inhibitor 1 .

Changes between baseline and month 6 were assessed with the paired samples t-test.

In overweight/obese women with PCOS, plasma PAI-1 levels at baseline correlated in univariate analysis with the BMI $(\mathrm{r}=0.300, \mathrm{p}=0.034)$, the $\mathrm{W}(\mathrm{r}=0.319$, $\mathrm{p}=0.024)$, serum LH levels $(\mathrm{r}=0.348, \mathrm{p}=0.013)$, the $\mathrm{LH} / \mathrm{FSH}$ ratio $(\mathrm{r}=0.386, \mathrm{p}=0.006)$, the FAI $(\mathrm{r}=0.347$, $\mathrm{p}=0.013)$, the mean ovarian volume $(\mathrm{r}=0.533, \mathrm{p}$ $<0.001)$ and the mean number of follicles in the ovaries $(r=0.340, p=0.016)$. In multivariate regression analysis, plasma PAI-1 levels were independently correlated with the BMI, serum $\mathrm{LH}$ levels and the mean ovarian volume $(\mathrm{p}=0.007, \mathrm{p}=0.002$ and $\mathrm{p}<0.001$, respectively), after adjusting for $\mathrm{W}, \mathrm{LH} / \mathrm{FSH}$ ratio, FAI and the mean number of follicles in the ovaries.

At baseline, overweight/obese women randomized to receive sibutramine had higher serum $\mathrm{LH}, \mathrm{T}$ and $17 \alpha$-OHP levels as well as greater FAI than women randomized to receive orlistat $(p=0.009, p=0.020$, $\mathrm{p}=0.011$ and $\mathrm{p}=0.037$, respectively). Other parameters did not differ at baseline between these two groups.

Changes in overweight/obese women with PCOS after 6 months of sibutramine or orlistat treatment are shown in Table 2. Both sibutramine and orlistat reduced the BMI, the $\mathrm{W}$ and the waist/hip ratio (WHR); these reductions did not differ between the two agents. In contrast, the effects of sibutramine and orlistat on the FAI were significantly different $(\mathrm{p}=0.027)$; sibutramine reduced the FAI $(\mathrm{p}=0.005)$, whereas orlistat had no significant effect. The effects of the two agents on the other circulating androgens were not significant. Both sibutramine and orlistat improved markers of IR (serum insulin levels, the glucose/insulin ratio and the indices HOMA-IR and QUICKI); this improvement did not differ between the two agents. However, the effects of sibutramine and orlistat on plasma PAI-1 levels were significantly different $(p=0.042)$ : sibutramine reduced plasma PAI-1 levels $(p<0.001)$, whereas orlistat had no significant effect.

In the total study population $(n=73)$, the reduction in plasma PAI-1 levels was greater in women with PCOS treated with metformin than in women treated with sibutramine or orlistat after adjusting for the change in BMI $(p=0.007)$.

\section{DISCUSSION}

To our knowledge, this is the first study that assessed the effects of metformin on circulating PAI-1 levels in normal weight women with PCOS. Three previous studies in overweight/obese women with PCOS reported a decline in circulating PAI-1 levels after treatment with metformin for 6 months, ${ }^{20-22}$ but two others did not show a significant change after treatment for 1.5 to 24 months. ${ }^{23,24}$ In normal weight women with PCOS, we observed a significant reduction in plasma PAI-1 antigen levels after treatment with metformin for 6 months. This decrease might be attributed to the reduction in IR and in the BMI, which are associated with elevated PAI-1 levels. ${ }^{6}$ Interestingly, in the three studies that reported a decline in PAI-1 levels after treatment with metformin there was no change in BMI, whereas markers of IR improved..$^{20-22}$ In contrast, IR did not change despite a reduction in BMI in a study that did not detect a change in PAI-1 levels after treatment with metformin. ${ }^{23}$ Therefore, the improvement of IR appears to contribute more 


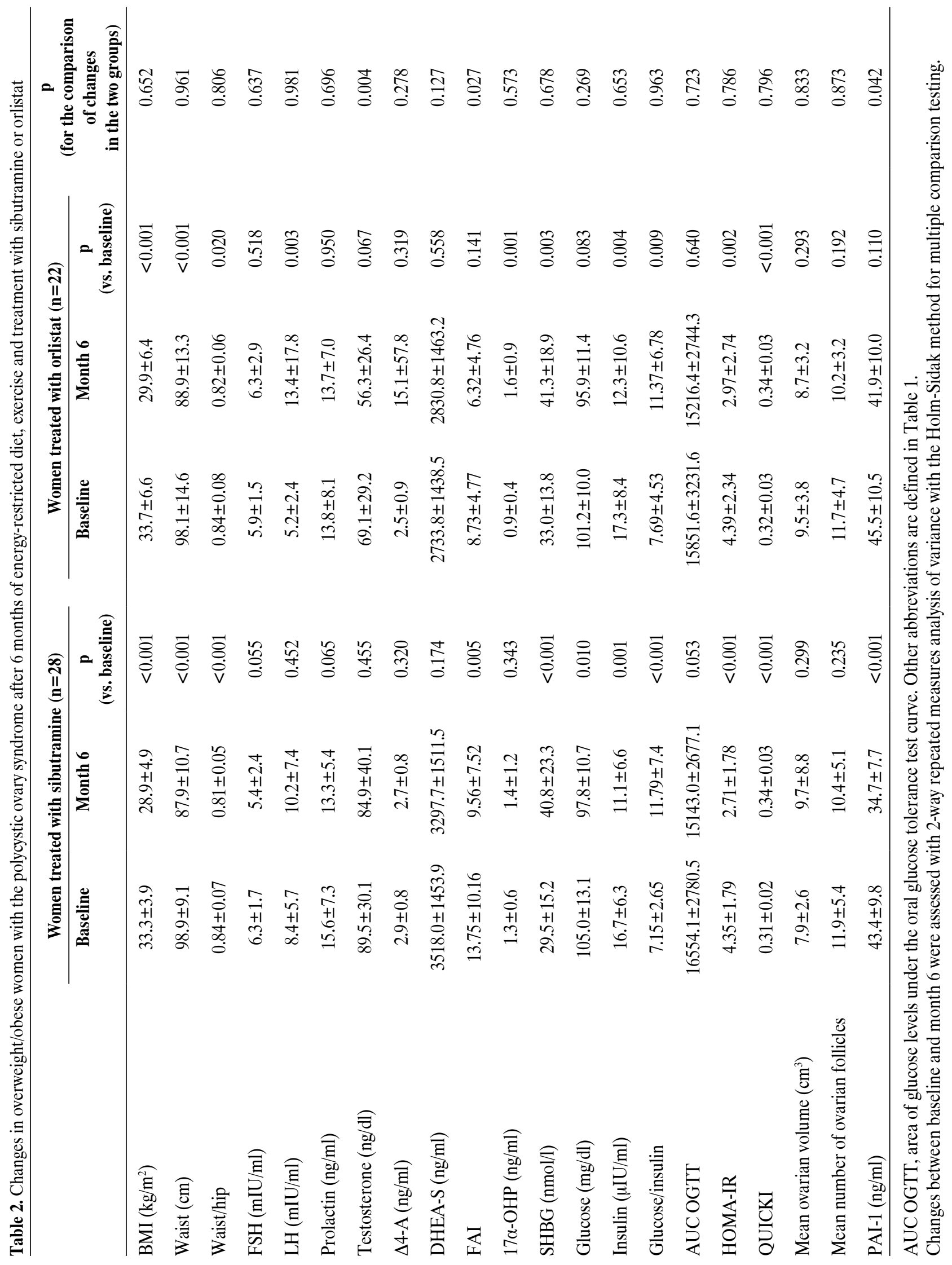


than weight loss to the reduction in PAI-1 levels during treatment with metformin. We also observed a reduction in circulating androgens after metformin treatment in agreement with previous studies. ${ }^{21,25}$ Some studies have suggested that hyperandrogenemia might be implicated in the increase in PAI-1 levels in women with PCOS. ${ }^{26,27}$ Therefore, the decline in circulating androgens during metformin treatment might also have played a role in the reduction in PAI-1 levels.

This is also the first study that evaluated the change in circulating PAI-1 levels in overweight/obese women with PCOS after low-calorie diet and exercise combined with either sibutramine or orlistat. A significant decrease in plasma PAI-1 levels was observed in patients treated with sibutramine, whereas orlistat had no significant effect. In agreement with previous studies, ${ }^{28-30}$ both sibutramine and orlistat induced a reduction in BMI, W and WHR and improved markers of IR. However, these beneficial effects did not differ between the two agents and therefore cannot explain the reduction in PAI-1 levels during sibutramine treatment. On the other hand, only sibutramine lowered the FAI, whereas orlistat did not affect the FAI or circulating androgens. Therefore, it appears that the reduction in hyperandrogenemia in women treated with sibutramine is implicated in the reduction in plasma PAI-1 antigen levels. Indeed, previous studies in overweight/obese women with PCOS reported an association between PAI-1 levels and hyperandrogenemia. ${ }^{5,31} \mathrm{We}$ also observed an independent correlation between plasma PAI-1 antigen levels and plasma LH levels, which stimulates ovarian androgen synthesis. ${ }^{32}$ On the other hand, in an early study in 9 obese women with PCOS, verylow-calorie diet (421 kcal/day) for 4 weeks followed by low-calorie diet (1000-1500 kcal/day) for 20 weeks reduced serum PAI-1 activity without affecting circulating androgens. ${ }^{12} \mathrm{~A}$ reduction in BMI and an improvement in markers of IR were also observed. ${ }^{12}$ However, the statistical power of this small study might have been too limited to detect a reduction in hyperandrogenemia. Indeed, larger studies reported a decrease in circulating androgens in obese women with PCOS following a low-calorie diet but did not measure PAI-1 levels. ${ }^{33}$

It is unclear why only sibutramine and not orlistat lowered circulating androgens in our study. Both obe- sity and IR are associated with hyperandrogenemia in women with PCOS. ${ }^{1,11}$ However, sibutramine and orlistat yielded comparable reductions in BMI, W, WHR and IR; therefore, these effects cannot explain the selective decrease in circulating androgens during sibutramine treatment. On the other hand, previous studies in obese women with PCOS have reported an amelioration of hyperandrogenemia during treatment not only with sibutramine ${ }^{29}$ but also with orlistat. ${ }^{28,30}$ In addition, orlistat reduced plasma PAI-1 activity in obese women without hyperandrogenemia. ${ }^{34}$ Clearly, additional studies are needed to elucidate the effects of different weight-loss strategies on androgen levels and whether these effects play a role in the reduction in circulating PAI-1 levels.

Since elevated PAI-1 levels and activity appear to be associated with increased risk for both T2DM and $C V D,{ }^{8,9}$ i.e. conditions that appear to be frequent in women with PCOS, the decrease in PAI-1 levels that was observed during metformin and sibutramine treatment might reduce the incidence of CVD and T2DM in this population. However, the present results remain to be confirmed or refuted in larger studies. More importantly, it should be mentioned that sibutramine was withdrawn in the European Union and the United States following the results of the Sibutramine Cardiovascular Outcome Trial (SCOUT), which showed increased cardiovascular morbidity in high-risk patients treated with sibutramine. ${ }^{35}$ Our study was designed and conducted before the publication of the results of the SCOUT trial. It is highly questionable whether the beneficial effects of sibutramine on obesity, IR, hyperandrogenemia and PAI-1 levels that were observed in women with PCOS justify its use in this relatively young and lower-risk population.

In conclusion, treatment with metformin lowers plasma PAI-1 antigen levels in normal weight women with PCOS. In overweight/obese women with the syndrome, sibutramine, but not orlistat, also reduces plasma PAI-1 antigen levels. In both normal weight and overweight/obese women with PCOS, the reduction in circulating androgens appears to be implicated in the decline in plasma PAI-1 antigen levels. More studies are needed to evaluate whether this decrease will translate into reduced risk for T2DM and CVD in women with PCOS. 


\section{REFERENCES}

1. Goodarzi MO, Dumesic DA, Chazenbalk G, Azziz R, 2011 Polycystic ovary syndrome: etiology, pathogenesis and diagnosis. Nat Rev Endocrinol 7: 219-231.

2. Dunaif A, Segal KR, Futterweit W, Dobrjansky A, 1989 Profound peripheral insulin resistance, independent of obesity, in polycystic ovary syndrome. Diabetes 38 : 1165-1174.

3. Morales AJ, Laughlin GA, Butzow T, et al, 1996 Insulin, somatotropic, and luteinizing hormone axes in lean and obese women with polycystic ovary syndrome: common and distinct features. J Clin Endocrinol Metab 81: 2854-2864.

4. Cesari M, Pahor M, Incalzi RA, 2010 Plasminogen activator inhibitor-1 (PAI-1): a key factor linking fibrinolysis and age-related subclinical and clinical conditions. Cardiovasc Ther 28: e72-e91.

5. Moran LJ, Hutchison SK, Meyer C, Zoungas S, Teede HJ, 2009 A comprehensive assessment of endothelial function in overweight women with and without polycystic ovary syndrome. Clin Sci (Lond) 116: 761-770.

6. Orio F, Jr., Palomba S, Cascella T, et al, 2004 Is plasminogen activator inhibitor-1 a cardiovascular risk factor in young women with polycystic ovary syndrome? Reprod Biomed Online 9: 505-510.

7. Tarkun I, Canturk Z, Arslan BC, Turemen E, Tarkun P, 2004 The plasminogen activator system in young and lean women with polycystic ovary syndrome. Endocr J 51: 467-472.

8. Meigs JB, O'donnell CJ, Tofler GH, et al, $2006 \mathrm{He}-$ mostatic markers of endothelial dysfunction and risk of incident type 2 diabetes: the Framingham Offspring Study. Diabetes 55: 530-537.

9. Smith A, Patterson C, Yarnell J, et al, 2005 Which hemostatic markers add to the predictive value of conventional risk factors for coronary heart disease and ischemic stroke? The Caerphilly Study. Circulation 112: 3080-3087.

10. Wild RA, Carmina E, Diamanti-Kandarakis E, et al, 2010 Assessment of cardiovascular risk and prevention of cardiovascular disease in women with the polycystic ovary syndrome: a consensus statement by the Androgen Excess and Polycystic Ovary Syndrome (AE-PCOS) Society. J Clin Endocrinol Metab 95: 2038-2049.

11. Norman RJ, Dewailly D, Legro RS, Hickey TE, 2007 Polycystic ovary syndrome. Lancet 370: 685-697.

12. Andersen P, Seljeflot I, Abdelnoor M, et al, 1995 Increased insulin sensitivity and fibrinolytic capacity after dietary intervention in obese women with polycystic ovary syndrome. Metabolism 44: 611-616.

13. Rotterdam ESHRE/ASRM-Sponsored PCOS consensus workshop group, 2004 Revised 2003 consensus on diagnostic criteria and long-term health risks related to polycystic ovary syndrome (PCOS). Hum Reprod 19: 41-47.
14. Koiou E, Tziomalos K, Katsikis I, et al, 2012 Weight loss significantly reduces serum lipocalin-2 levels in overweight and obese women with polycystic ovary syndrome. Gynecol Endocrinol 28: 20-24.

15. Koiou E, Tziomalos K, Dinas K, et al, 2011 The effect of weight loss and treatment with metformin on serum vaspin levels in women with polycystic ovary syndrome. Endocr J 58: 237-246.

16. Piouka A, Farmakiotis D, Katsikis I, et al, 2009 AntiMullerian hormone levels reflect severity of PCOS but are negatively influenced by obesity: relationship with increased luteinizing hormone levels. Am J Physiol Endocrinol Metab 296: E238-E243.

17. Carter GD, Holland SM, Alaghband-Zadeh J, et al, 1983 Investigation of hirsutism: testosterone is not enough. Ann Clin Biochem 20 (Pt 5): 262-263.

18. Matthews DR, Hosker JP, Rudenski AS, et al, 1985 Homeostasis model assessment: insulin resistance and beta-cell function from fasting plasma glucose and insulin concentrations in man. Diabetologia 28: 412-419.

19. Katz A, Nambi SS, Mather K, et al, 2000 Quantitative insulin sensitivity check index: a simple, accurate method for assessing insulin sensitivity in humans. $\mathrm{J}$ Clin Endocrinol Metab 85: 2402-2410.

20. Palomba S, Orio F, Jr., Falbo A, et al, 2005 Plasminogen activator inhibitor 1 and miscarriage after metformin treatment and laparoscopic ovarian drilling in patients with polycystic ovary syndrome. Fertil Steril 84: 761765.

21. Tan BK, Adya R, Chen J, et al, 2009 Metformin decreases angiogenesis via NF-kappaB and Erk1/2/Erk5 pathways by increasing the antiangiogenic thrombospondin-1. Cardiovasc Res 83: 566-574.

22. Teede HJ, Meyer C, Hutchison SK, et al, 2010 Endothelial function and insulin resistance in polycystic ovary syndrome: the effects of medical therapy. Fertil Steril 93: 184-191.

23. Agarwal N, Rice SP, Bolusani H, et al, 2010 Metformin reduces arterial stiffness and improves endothelial function in young women with polycystic ovary syndrome: a randomized, placebo-controlled, crossover trial. J Clin Endocrinol Metab 95: 722-730.

24. Glueck CJ, Wang P, Fontaine R, Tracy T, Sieve-Smith L, 1999 Metformin-induced resumption of normal menses in 39 of 43 (91\%) previously amenorrheic women with the polycystic ovary syndrome. Metabolism 48: 511519.

25. Orio F, Jr., Palomba S, Cascella T, et al, 2005 Improvement in endothelial structure and function after metformin treatment in young normal-weight women with polycystic ovary syndrome: results of a 6-month study. J Clin Endocrinol Metab 90: 6072-6076.

26. Carmassi F, De NF, Fioriti R, et al, 2005 Insulin resistance causes impaired vasodilation and hypofibrinolysis in young women with polycystic ovary syndrome. 
Thromb Res 116: 207-214.

27. Manneras-Holm L, Baghaei F, Holm G, et al, 2011 Coagulation and fibrinolytic disturbances in women with polycystic ovary syndrome. J Clin Endocrinol Metab 96: 1068-1076.

28. Diamanti-Kandarakis E, Katsikis I, Piperi C, Alexandraki K, Panidis D, 2007 Effect of long-term orlistat treatment on serum levels of advanced glycation endproducts in women with polycystic ovary syndrome. Clin Endocrinol (Oxf) 66: 103-109.

29. Florakis D, Diamanti-Kandarakis E, Katsikis I, et al, 2008 Effect of hypocaloric diet plus sibutramine treatment on hormonal and metabolic features in overweight and obese women with polycystic ovary syndrome: a randomized, 24-week study. Int J Obes (Lond) 32: 692-699.

30. Panidis D, Farmakiotis D, Rousso D, et al, 2008 Obesity, weight loss, and the polycystic ovary syndrome: effect of treatment with diet and orlistat for 24 weeks on insulin resistance and androgen levels. Fertil Steril
89: 899-906.

31. Lindholm A, Bixo M, Eliasson M, et al, 2010 Tissue plasminogen activator and plasminogen activator inhibitor 1 in obese and lean patients with polycystic ovary syndrome. Gynecol Endocrinol 26: 743-748.

32. Glintborg D, Andersen M, 2010 An update on the pathogenesis, inflammation, and metabolism in hirsutism and polycystic ovary syndrome. Gynecol Endocrinol 26: 281-296.

33. Stamets K, Taylor DS, Kunselman A, et al, 2004 A randomized trial of the effects of two types of shortterm hypocaloric diets on weight loss in women with polycystic ovary syndrome. Fertil Steril 81: 630-637.

34. Rissanen $P$, Vahtera E, Krusius T, Uusitupa M, Rissanen A, 2001 Weight change and blood coagulability and fibrinolysis in healthy obese women. Int J Obes Relat Metab Disord 25: 212-218.

35. James WP, Caterson ID, Coutinho W, et al, 2010 Effect of sibutramine on cardiovascular outcomes in overweight and obese subjects. N Engl J Med 363: 905-917. 